\title{
Programa de intervenção nutricional associado à atividade física: discurso de idosas obesas
}

\author{
Nutritional intervention program associated with physical \\ activity: discourse of obese elderly women
}

Christiane Leite Cavalcanti ${ }^{1}$

Maria da Conceição Rodrigues Gonçalves ${ }^{1}$

Alessandro Leite Cavalcanti ${ }^{2}$

Solange de Fátima Geraldo da Costa ${ }^{1}$

Luiza Sonia Rios Asciutti ${ }^{1}$

${ }^{1}$ Universidade Federal da Paraíba. Campus Universitário, Cidade Universitária. 58000-000 João Pessoa PB. chris tiane2006@hotmail.com

${ }^{2}$ Universidade Estadual da

Paraíba.

\begin{abstract}
Obesity is a complex nutritional problem with social and psychological dimensions, which affects individuals of all ages. In addition to presenting risk factors for some diseases, obesity may interfere with the quality of life of the elderly. This qualitative study of an exploratory nature investigated the discourse of obese elderly women regarding their participation in a nutritional intervention program associated with physical activity. Eighteen obese elderly women attending Centers of Reference and Citizenship in the city of João Pessoa, state of Paraiba, Brazil were enlisted in the study. Interviews using a series of questions that addressed the core issue pertaining to the scope of the study was the technique used for data collection. The data obtained were analyzed by the collective subject discourse (CSD) technique. The following central ideas emerged from CSD: changes in lifestyle, in eating habits, in health and in selfesteem. The core ideas reflected the value attributed to the aforesaid program for promotion of health of the group of elderly women who participated in the study. It is hoped that this research might elicit further investigation in the area of health of the elderly.
\end{abstract}

Key words Qualitative research, Health of the elderly, Obesity, Motor activity
Resumo A obesidade é um problema nutricional complexo, com dimensões sociais e psicológicas, que afeta indivíduos de todas as idades. Além de apresentar fatores de risco para algumas doenças, pode interferir na qualidade de vida do idoso. Este estudo exploratório de natureza qualitativa investigou o discurso de idosas obesas quanto à participação em um programa de intervenção nutricional associado à atividade física. Participaram da pesquisa dezoito idosas obesas, inseridas em Centros de Referência e Cidadania do município de João Pessoa (PB). Para a coleta de dados, foi utilizada a técnica da entrevista a partir de um roteiro que contemplou a questão norteadora pertinente ao objetivo da investigação. Os dados obtidos foram analisados mediante a técnica de discurso do sujeito coletivo (DSC). Do DSC emergiram as seguintes ideias centrais: mudanças no estilo de vida, no hábito alimentar, na saúde e na autoestima. As ideias centrais refletem a valorização do referido programa para a promoção da saúde do grupo de idosas participantes do estudo. Espera-se que esta proposta possa subsidiar novas investigações na área da saúde do idoso.

Palavras-chave Pesquisa qualitativa, Saúde do idoso, Obesidade, Atividade fisica 


\section{Introdução}

O envelhecimento constitui um processo biológico de declínio das capacidades físicas, psicológicas e comportamentais de maneira irreversível, individual e universal. Envelhecer é tornar-se mais vulnerável às agressões do meio interno e externo e, portanto, caracteriza-se por uma maior suscetibilidade tanto celular quanto tecidual, orgânica e nos aparelhos e sistemas ${ }^{1}$.

No Brasil, o processo de envelhecimento vem ocorrendo em um ritmo acelerado, visto que, segundo a Organização Mundial da Saúde, até o ano de 2025 o país será classificado como a sexta população do mundo em idosos, correspondendo a mais de 32 milhões de pessoas com 60 anos ou mais de idade. Este crescimento populacional é o mais acelerado no mundo e só comparável ao do México e da Nigéria².

A Organização Mundial da Saúde considera idosos, nos países em desenvolvimento, aqueles que atingiram a idade de 60 anos, enquanto nos países desenvolvidos o recorte etário é de 65 anos. A Legislação Brasileira (Lei no 8.842/94, em seu artigo $2^{\circ}$, parágrafo único) refere que são consideradas idosas as pessoas maiores de 60 anos, de ambos os gêneros, sem distinção de cor, etnia e ideologia ${ }^{3}$.

As modificações ocorridas no cenário demográfico brasileiro, resultando em um aumento substancial do número de pessoas idosas, são acompanhadas por modificações no perfil epidemiológico e nutricional da população. Nesse contexto, predominam as doenças crônicas não transmissíveis (DCNT), e a obesidade é considerada uma doença integrante desse grupo ${ }^{4}$.

O excesso de peso atinge cerca de $1 / 3$ da população adulta brasileira e apresenta uma tendência crescente nas últimas décadas. Há uma prevalência maior de obesidade entre as mulheres, inclusive entre idosos. Em ambos os gêneros, seu auge ocorre entre 45 e 64 anos. $O$ excesso de peso corporal assume uma importância ainda maior entre os idosos, considerando que entre estes se verifica uma frequência elevada de enfermidades. Neste caso, o excesso de peso poderia potencializar os efeitos dessas enfermidades ${ }^{5}$.

É relevante mencionar que o excesso de peso e a obesidade levam a distúrbios das condições de saúde do organismo, tais como: diabetes mellitus, hipertensão, dislipidemias, certos tipos de câncer, sendo relacionados também à ocorrência de eventos cardiovasculares ${ }^{6}$, além de outras doenças que interferem na qualidade de vida do indivíduo, especialmente no idoso ${ }^{7}$. Essas alterações podem ser representadas por distúrbios psicológicos, sociais e aumento do risco de morte prematura ${ }^{8}$.

Os distúrbios psicológicos, incluindo depressão, distúrbios alimentares, imagem corporal distorcida e baixa autoestima, estão associados ao sobrepeso e à obesidade ${ }^{9}$. As prevalências de ansiedade e depressão são de três a quatro vezes mais altas entre indivíduos obesos. Além disso, indivíduos obesos são estigmatizados e sofrem discriminação social ${ }^{10}$.

Sabe-se que a obesidade tem impacto relevante na longevidade, e qualquer iniciativa com vistas à redução de peso traz benefícios que podem ser observados em longo prazo, e que tendem a ser maiores quanto mais precoces forem essas intervenções. Isto não apenas para os graus maiores de obesidade, mas inclusive para os que se encontram na faixa de sobrepeso ${ }^{11}$.

A elevada prevalência de indivíduos idosos com excesso de peso e obesidade evidencia a necessidade de elaboração de medidas específicas direcionadas para essa faixa etária, em prol da efetividade nas intervenções, como por exemplo programas de intervenção nutricional associada à atividade física.

Cumpre assinalar que a saúde e a qualidade de vida dos idosos, mais que em outros grupos etários, sofrem a influência de múltiplos fatores físicos, psicológicos, sociais e culturais. Assim, avaliar e promover a saúde do idoso significa considerar variáveis de distintos campos do saber, numa atuação interdisciplinar e multidimensional. Portanto, o objetivo deste trabalho foi investigar o discurso de idosas obesas quanto à participação em um programa de intervenção nutricional associado à atividade física.

\section{Metodologia}

Trata-se de um estudo exploratório de natureza qualitativa. A investigação foi desenvolvida com idosas obesas inseridas em Centros de Referência e Cidadania (CRC) pertencentes à Secretaria do Desenvolvimento Social da Prefeitura de João Pessoa (PB). Esses centros constituem-se em espaços privilegiados voltados à valorização dos idosos, com o desenvolvimento de atividades de lazer, trabalhos manuais, noções de saúde e o exercício da cidadania. Atualmente, existem dez CRCs, e considerando-se a homogeneidade dos centros no que diz respeito às atividades desenvolvidas e ao nível socioecônomico das participantes, foram sorteados de forma aleatória simples três centros, distribuídos em diferentes bairros do referido município. 
Para a seleção das participantes do estudo, foram adotados os seguintes critérios: apresentar sobrepeso ou obesidade; ter participado e concluído o programa de intervenção nutricional com duração de 12 semanas, o qual envolveu dois distintos grupos: Grupo Dieta (GD), somente intervenção nutricional, e Grupo Dieta e Atividade Física (GDAF), intervenção nutricional aliada à prática de atividade física, e que aceitassem participar da investigação proposta por meio da assinatura do Termo de Compromisso Livre e Esclarecido. Com base nesses critérios, participaram da presente pesquisa dezoito idosas, com idades entre 60 e 76 anos. Com relação ao estado nutricional, nove possuíam sobrepeso e nove apresentavam obesidade, estando as participantes assim distribuídas: GD - cinco com sobrepeso e quatro apresentando obesidade; e GDAF - quatro com sobrepeso e cinco apresentando obesidade.

Todas as 18 participantes, após avaliação nutricional inicial, foram orientadas a cumprir uma dieta hipocalórica, com redução de quinhentas calorias por dia. Para a elaboração das dietas, foi utilizado o software para acompanhamento nutricional Santé 2.0, elaborado pelo Núcleo de Estudos e Tecnologia em Engenharia Biomédica juntamente com o Departamento de Nutrição da Universidade Federal da Paraíba. A dieta compreendia a ingestão de calorias oriundas de carboidratos (55\%-60\%), lipídeos (20\%-25\%) e proteínas (15\%-20\%) distribuídas em cinco a seis refeições/dia, contemplando ainda aumento do aporte de fibras e redução da ingestão de gorduras saturadas, colesterol, carboidratos simples e sódio. Além da dieta, os indivíduos do GDAF foram orientados à prática de atividade física, sendo escolhido o exercício aeróbico do tipo ginástica sob supervisão. Foram indicadas duas sessões por semana, sendo cada sessão com 45 minutos de duração, distribuídos da seguinte forma: cinco minutos de aquecimento, trinta minutos de ginástica e dez minutos de alongamento. Após o período da coleta de dados, as componentes do GD foram orientadas e motivadas a participar da atividade física oferecida pelos Centros, visando à adoção de um estilo de vida ativo.

Para a apreensão do fenômeno a ser investigado, utilizou-se a técnica de entrevista, a partir de um roteiro contendo uma questão norteadora pertinente ao objetivo da pesquisa. As entrevistas foram coletadas no mês de agosto de 2007, após o término do programa de intervenção nutricional. Para apreensão do material empírico, foi usado o sistema de gravação em fita cassete, por possibilitar a captação fidedigna do discurso das participantes. Como ponto de partida das entrevistas realizadas, foi formulada a seguinte questão norteadora: que mudanças a senhora observou após o programa de intervenção nutricional aliado à prática de atividade física?

As entrevistas foram realizadas nos próprios centros, em salas de atendimento individual, tendo duração de vinte minutos cada uma. Ao final de cada entrevista, a idosa era convidada para ouvir a gravação, como forma de garantir a fidedignidade dos discursos colhidos.

Os dezoito discursos constituem o material de análise deste estudo, sendo trabalhados da seguinte forma: no primeiro momento, os discursos foram transcritos na íntegra, de acordo com a sequência de sua realização. $\mathrm{Na}$ etapa seguinte, fez-se uma leitura individualizada dos discursos, com o objetivo de se ter uma visão mais ampla de cada um, buscando os pontos de convergência que surgiam entre eles, na perspectiva do fenômeno pesquisado. Seguindo essa trajetória, buscou-se refletir com maior profundidade sobre os significados que as participantes da pesquisa atribuíram ao fenômeno investigado, procurando apreendê-lo em sua essência.

$\mathrm{Na}$ análise dos dados, foi empregada a técnica de análise do discurso do sujeito coletivo (DSC), proposta por Lefévre e Lefévre ${ }^{12}$. Esta técnica permite resgatar o discurso sobre um determinado tema, em um dado universo. O discurso do sujeito coletivo para os referidos autores implica um radical rompimento com a lógica qualitativo-classificatória, na medida em que se busca resgatar o discurso como signo de conhecimentos dos próprios discursos ${ }^{12}$.

A análise do discurso do sujeito coletivo das participantes envolveu as seguintes etapas: seleção das expressões-chave de cada discurso individual, obtidas para cada questão proposta para o estudo; identificação das ideias centrais que cada um dos indivíduos apresenta em seu discurso e as expressões-chave para cada resposta de uma dada pergunta, formando a síntese do conteúdo dessas expressões; agrupamento das ideias centrais semelhantes ou complementares, envolvendo as mesmas respostas de um questionamento proposto para a pesquisa, transcrevendo-se literalmente os termos utilizados pelas participantes do estudo e construção do discurso-síntese, ou discurso do sujeito coletivo, através do agrupamento das ideias centrais semelhantes. Para a construção do DSC, várias ideias centrais e várias expressões-chave foram reunidas em um só discurso, como se todas tivessem sido proferidas por um mesmo indivíduo. 
Seguindo os preceitos éticos vigentes, estabelecidos na Resolução n ${ }^{\circ}$ 169/96 do Conselho Nacional de Saúde, esta pesquisa foi aprovada pelo Comitê de Ética em Pesquisa do Centro de Ciências da Saúde da Universidade Federal da Paraíba.

\section{Resultados e discussão}

As respostas obtidas da questão norteadora permitiram construir quatro ideias centrais: mudanças no estilo de vida, mudança nos hábitos alimentares, mudanças na saúde e mudanças na autoestima. A seguir, são apresentadas as ideias centrais e os discursos do sujeito coletivo, construído a partir da percepção das questões propostas para o estudo.

\section{Apresentando \\ o discurso do sujeito coletivo}

Na primeira ideia central (Quadro 1), o discurso do sujeito coletivo das participantes inseridas no estudo expressa, de forma clara, a mudança no estilo de vida, efetivada pelo cuidado com a alimentação e a prática da ginástica. $\mathrm{O}$ reconhecimento da importância das orientações propostas pelo programa foi muito enfatizado pelas participantes da pesquisa, conforme está assinalado nos seguintes trechos:

Eu vou continuar a seguir as orientações, é uma mudança para a vida toda.

Hoje eu sou outra pessoa, depois do programa. Hoje eu cuido da alimentação e faço ginástica, coisa que eu não fazia antes.

Esses relatos expressam o reconhecimento das idosas entrevistadas no que se refere à importância de uma alimentação saudável e da prática de atividade física para a manutenção da saúde e promoção de bem-estar. Nesse sentido, Burdel-
Marchassom e Traissac ${ }^{13}$ ressaltam que toda proposta terapêutica para a obesidade deve ser realista, maleável e ter como meta principal uma melhor qualidade de vida, com ou sem redução de peso.

Os hábitos alimentares e a prática de atividade física promovem efeitos positivos em várias funções fisiológicas e vêm sendo discutidos, em estudos de revisões, como elementos fundamentais na melhora da saúde e qualidade de vida dos indivíduos, especialmente nos idosos ${ }^{14}$.

Dentre as intervenções, destaca-se o papel do exercício físico, discutido em várias revisões e estudos, que atribuem à prática regular de atividade física - mesmo se iniciada após os 60 anos maior longevidade, efeito benéfico independente nas comorbidades da obesidade e benefícios psicológicos, como melhora da afetividade, da socialização e da autoestima em idosos ${ }^{2}$.

A atividade física é um componente importante no tratamento da obesidade, e a combinação de dieta mais exercício físico proporciona perdas de peso mais eficientes do que somente a dieta isola$\mathrm{da}^{6}$. As intervenções que mais afetam a qualidade de vida no envelhecimento estão centradas na eliminação do fumo, no aumento da atividade física habitual e na melhora dos padrões nutricionais ${ }^{15}$.

A participação do idoso em programas estruturados de atividade física proporciona uma maximização de contatos sociais, favorecendo melhorias na satisfação com a vida e redução da solidão. O idoso fisicamente ativo é capaz de superar as exigências impostas pela atividade física (autoeficácia), resgatar a autovalorização e a autoconfiança, o que irá interferir positivamente na autopercepção da imagem corporal. O resgate desses aspectos, promovido pela prática de atividade física, melhora a forma como o indivíduo lida com seu corpo e pode refletir em outros comportamentos relacionados ao bem-estar, como a alimentação e o convívio social.

Quadro 1. Ideia central 1 e discurso do sujeito coletivo das participantes.

\begin{tabular}{|l|}
\hline 1a ideia central \\
\hline Mudanças no estilo de vida \\
\hline Discurso do sujeito coletivo \\
\hline Eu não tive nenhuma dificuldade em seguir a alimentação, achei muito boa e fácil de fazer. Eu vou continuar \\
a seguir as orientações, é uma mudança para a vida toda. Não tive problema nenhum em fazer a ginástica, ao \\
contrário, eu adorei fazer ginástica. Achei muito bom mesmo. Toda vez que tem aula de ginástica, eu fico \\
animada. Gostei da dieta e da ginástica e pretendo seguir daqui pra frente. Hoje eu sou outra pessoa, depois \\
do programa. Hoje eu cuido da alimentação e faço ginástica, coisa que eu não fazia antes. Mudou muita coisa \\
em minha vida. Eu achei que foi muito bom o que foi feito pra mim.
\end{tabular}


O DSC das participantes da pesquisa destaca a motivação das idosas para a realização de atividades físicas, por proporcionarem alterações positivas nos estados de ânimo, na autoestima, na autoeficácia, obtendo recursos pessoais para poder encarar as situações de estresse do dia a dia.

Na segunda ideia central (Quadro 2), o discurso do sujeito coletivo das idosas participantes da investigação demonstram que todas as ações educativas voltadas para as mudanças dos hábitos alimentares foram efetivas, como revela o seguinte trecho do DSC:

Depois do programa, mudei os hábitos de comer, os horários exatos, porque eu comia exageradamente, aí eu tô moderando, né? Mudou e eu creio que devia, porque eu estava comendo o que não devia, aí eu como o que devo e em poucas quantidades.

Esse trecho do DSC deixa transparecer o valor das ações educativas de alimentação e nutrição. Estas não devem se limitar a informar e elevar os conhecimentos sobre alimentação e nutrição; devem, além disso, estimular a adoção de atitudes e práticas alimentares adequadas. Fazem-se necessários o estímulo à mudança de comportamento da população de risco e a divulgação de informações sobre medidas de prevenção e controle do sobrepeso e da obesidade.

O processo de envelhecimento é determinado por diversos fatores, entre os quais se podem citar os de natureza genética, que não são passíveis de intervenção, e os de natureza ambiental, sobre os quais se pode agir ${ }^{16}$. Entre estes últimos, encontra-se a alimentação, que exerce papel fundamental na promoção, na manutenção e na re- cuperação da saúde, desde que seja nutricionalmente adequada.

A existência humana depende da interação entre o homem e o meio em que ele vive, e uma dessas interações se relaciona com os hábitos alimentares. Quando preponderam os bons hábitos, ocorre uma vida longa e saudável; os maus hábitos, ao contrário, podem determinar uma vida mais breve e com manifestações de diversas doenças ${ }^{11}$.

Diversos autores relatam que o principal fator que determina o potencial de longevidade é a nutrição adequada. Isto faz crer que o tipo de alimentação pode estar diretamente associado à qualidade de vida do indivíduo, pois uma alimentação adequada pode diminuir o risco de incidência de certas doenças. Os hábitos dietéticos inadequados apresentam-se como um importante fator de risco para o surgimento de doenças crônicas em idosos ${ }^{17}$. Nesse sentido, há a necessidade de intervenções nutricionais direcionadas a esse grupo etário para esclarecer a importância da alimentação saudável no controle do peso corporal, a fim de minimizar a ocorrência de doenças em prol de melhor saúde e qualidade de vida.

O DSC das entrevistadas participantes da pesquisa revela aspectos positivos relativos a melhorias na saúde, como evidenciado no Quadro 3. Portanto, é inegável a relevância do programa de intervenção nutricional associado à prática de atividade física na promoção da saúde das idosas participantes, que se traduz em uma sensação de maior disposição nas atividades diárias, como

Quadro 2. Ideia central 2 e discurso do sujeito coletivo das participantes.

\begin{tabular}{l} 
2a ideia central \\
Mudanças no hábito alimentar \\
\hline Discurso do sujeito coletivo \\
\hline Depois do programa, mudei os hábitos de comer, os horários exatos, porque eu comia exageradamente, aí eu \\
tô moderando, né? Mudou e eu creio que devia, porque eu estava comendo o que não devia, aí eu como o que \\
devo e em poucas quantidades. Olha, aprendi muita coisa no programa. Eu sei as coisas que tem que comer e \\
as que ofende. Comer pouco, mastigar bastante e comer assim, seis vezes ao dia, tudo pouquinho. Tomar café \\
da manhã, lanchar de nove ou dez horas, 12 horas almoçar e de tarde lanchar um pouquinho também. Eu não \\
posso comer muita massa, né? Diminuí mais a comida, logo eu não gostava muito de comer pão, essas coisas. \\
Ass vezes, uma torradinha, um biscoitinho. Eu como mais assim, uma fruta à tarde. A massa eu como na parte \\
da manhã, um pouquinho. Um pão integral eu como uma banda. E a janta também, ficou macaxeira um \\
pedacinho, ficou cuscuz um pedacinho também, ficou uma papa com leite desnatado. Quando eu vou dormir, \\
eu tomo um suquinho de maracujá, eu chupo uma laranja ou duas, eu tomo um copo de leite com adoçante. Eu \\
como de tudo, toda fruta, toda verdura, só que eu resumo as horas de comer e as quantidades. Aprendi a comer \\
com o programa. Ainda tô com o papel em casa, da dieta.
\end{tabular}


andar, levantar-se, dormir, bem como a melhora no quadro de algumas doenças crônicas evidenciada nos depoimentos das participantes do estudo. O DSC das participantes do estudo deixa transparecer que havia uma diminuição acentuada da condição física, tornando a participação em atividades rotineiras difícil de iniciar ou de manter por tempo suficiente, devido ao cansaço e à limitação da liberdade dos movimentos.

O DSC revela também que as participantes obtiveram melhoras na saúde e, consequentemente, na qualidade de vida. Viver com qualidade é ter consciência do próprio comportamento e autocrítica que permitam avaliar as consequências do estilo de vida na relação consigo e com o outro ${ }^{15}$. Entre a consciência da necessidade da mudança de comportamento e sua real efetivação, estabelece-se uma longa distância. Mudar o comportamento e alterar hábitos arraigados prejudiciais à saúde e à qualidade de vida não se fazem por mágica ou pela simples vontade de mudar; é preciso ter disciplina, persistência, determinação, e incluem também o equilíbrio entre os estados físico, funcional, psicológico, social e os fatores econômicos. Em relação à perda de peso, mesmo não sendo o objetivo deste trabalho apresentar dados quantitativos, apenas para confirmar o discurso das idosas foram verificadas mudanças nas medidas antropométricas para os grupos GD (redução média de 1,4 kg no peso, $2,0 \mathrm{~cm}$ na cintura e $1,8 \mathrm{~cm}$ no quadril) e GDAF (redução média de 1,7 kg no peso, $2,7 \mathrm{~cm}$ na cintura e $1,7 \mathrm{~cm}$ no quadril).

Com relação às propostas de controle da obesidade, a maioria delas parece conduzir ao equilíbrio da ingestão energética somado ao aumento da atividade física, sem, no entanto, haver consenso sobre os tipos, duração e níveis de exercício e de dieta mais adequados às diversas situações. Nesse contexto, a principal etapa do tratamento da obesidade deve consistir numa reestruturação do comportamento do indivíduo, em busca de um estilo de vida saudável. Essas mudanças, uma vez atingidas, diminuem também os fatores de risco associados à obesidade ${ }^{11}$.

Na quarta ideia central (Quadro 4), o discurso do sujeito coletivo das idosas envolvidas na investigação expressa de forma bastante clara a elevação da autoestima, uma vez que o reconhecimento de seu novo visual, ou seja, seu corpo,

Quadro 3. Ideia central 3 e discurso do sujeito coletivo das participantes.

3a ideia central

Mudanças relacionadas à saúde

Discurso do sujeito coletivo

Mudou muita coisa, eu emagreci. Eu fiquei mais maneira, que eu era um pouco pesada, até na cama pra mim virar, eu tinha que embolar. Eu sou diabética, e a glicose, ela já foi pra 300, já foi pra 304 e eu fiz esse mês e ela tá em 221, aí meu colesterol também baixou, que era 200 e pouco, ficou 166. Eu durmo mais, porque eu não dormia, passava a noite todinha sem dormir, e agora eu tô dormindo bastante. Deito e só no outro dia. Eu fiquei mais leve, fiquei com mais vontade de fazer as coisas. Porque no começo, assim, eu sentia muito a gordura, eu cansava, sabe? E agora eu não tô cansando mais, né? Já é uma coisa boa. Mudou muita coisa, eu tô me sentindo mais levezinha, estou bem melhor, né? Tô me sentindo melhor, me sinto mais leve, com mais vontade de trabalhar, mais disposta.

Quadro 4. Ideia central 4 e discurso do sujeito coletivo das participantes.

$4^{\text {a }}$ ideia central

Mudanças na autoestima

Discurso do sujeito coletivo

Uma roupa que eu não vestia, uma calça que não cabia, eu já visto direitinho, eu me sinto muito bem. Eu gostei muito. Mudou meu peso, né? Eu tava com $69 \mathrm{~kg}$. Aí, quando eu cheguei aqui, que você me pesou, eu tava com $65 \mathrm{~kg}$, já tinha perdido $4 \mathrm{~kg}$. Aí, eu perdi mais 1,4 kg. Mudou tudo, foi tudo de bom. Então, eu me sinto melhor. Porque eu diminuí mais o peso. Porque perder uns quilos, ficar bem maneirinha, a roupa caber bem, não existe melhor. Eu estou feliz. Eu perdi $3 \mathrm{~kg}$. 
gera satisfação. Após a perda de peso, observase uma sensação de maior disposição e leveza, trazendo um bem-estar físico e emocional.

O DSC deixa transparecer que o bem-estar emocional revela-se como fator primordial da autoestima, ou seja, o próprio julgamento feito através de crenças e atitudes que as participantes inseridas na pesquisa têm em relação a si próprias e ao pensamento de outros com relação às suas capacidades. Nessa perspectiva, pessoas que adquiriram hábitos novos por meio de exercícios físicos e atividades sociais melhoraram sua autoestima e sua autoimagem ${ }^{18}$.

A autoestima e a autoimagem fazem parte do autoconhecimento. Quando a autoimagem encontra-se em equilíbrio, é possível ver com outros olhos o próprio corpo, e isso estimula a vitalidade, dá prazer, dá alegria, portanto, torna os idosos mais capacitados a perceberem seu espaço social para delimitá-lo de acordo com suas necessidades. Essa autoimagem inclui cuidados com a estética, sua valorização pessoal, sua bele$\mathrm{za}$, que exerce sobre as pessoas o domínio do encantamento, da vitalidade, da alegria, que abrange também sua aparência física, inclusive o seu peso. O peso é inevitavelmente vinculado à autoimagem ${ }^{19}$, desse modo proporcionando o bem-estar do ser idoso.

O conceito de bem-estar é semelhante ao de saúde, ou seja, estado de completo bem-estar físico, mental e social, e não meramente a ausência de doença. No entanto, todo bem-estar inclui uma abordagem consciente e deliberada do estado avançado de saúde física, psicológica e espiritual, também caracterizado por ser dinâmico e flutuante. O bem-estar é indicado pela capacidade que uma pessoa tem de realizar melhor suas potencialidades e por ajustar-se e adaptar-se às várias situações e, por fim, pelo relato de sensação de bem-estar, seguido de um sentimento de que todas as coisas se completam ${ }^{19}$.

$\mathrm{Na}$ terceira idade, ainda que as capacidades fisiológicas e o desempenho de esforço estejam certamente abaixo daqueles dos jovens, o idoso pode mudar com sucesso padrões de comportamento, de alimentação e de atividade, desde que motivado. A autonomia ajuda a eliminar barreiras sociais e culturais e permite com que a pessoa tenha uma visão positiva da vida, condição que a predisporá a novas buscas para o seu bem-estar, levando-a mesmo a mudar seu estilo de vida. Esse bem-estar pode estar intimamente relacionado com o bem-estar físico e geral da pessoa ${ }^{19}$.

Após o estudo com as idosas participantes do programa de intervenção nutricional associado à prática de atividade física, pode-se afirmar que a obesidade é uma dificuldade em todos os aspectos essenciais para a manutenção de uma boa qualidade de vida. Considerando o DSC e a literatura estudada, o sucesso da perda de peso se respalda na mudança dos hábitos alimentares, na prática da atividade física e na mudança comportamental.

\section{Considerações finais}

Os estudos qualitativos são de grande relevância para os profissionais de saúde que lidam com o cuidar, com a comunicação e com a interação entre as pessoas. Com relação às mudanças no estilo de vida, observou-se que as participantes aderiram à dieta prescrita e incorporaram a atividade física em suas vidas, podendo, assim, retardar muitos problemas e sintomas associados ao processo de envelhecimento, além de prolongar a saúde e o bem-estar nos anos seguintes. No que concerne às mudanças relacionadas aos hábitos alimentares, o DSC das idosas mostrou que é possível corrigir erros alimentares, buscando melhor qualidade de vida. Na promoção do bemestar físico, o DSC demonstrou que a mudança nos hábitos alimentares e na atividade física promoveu maior disposição para o autocuidado, bem como para o desenvolvimento de atividades ora impedidas pela obesidade.

Com relação à elevação da autoestima, observou-se que, a partir do programa de intervenção nutricional associado à atividade física, muitos ganhos foram concretizados, como vestir roupas de sua preferência, leveza nas atitudes diárias, além de outros atributos que contribuem para que a idosa se sinta bem consigo, aumentando assim o bem-estar psicológico. O DSC evidencia também que são indissociáveis o físico e o psicológico e que os dois mantêm dependência um em relação ao outro. Por isso, a integralidade "corpo e mente" deve ser considerada pelo profissional de saúde. 


\section{Colaboradores}

CL Cavalcanti trabalhou na coleta de dados, na interpretação dos resultados e na redação do artigo; MCR Gonçalves e SFG Costa, no delineamento metodológico, análise, discussão e interpretação dos resultados; AL Cavalcanti, na interpretação dos resultados, redação e revisão final do artigo; LSR Asciutti, no delineamento metodológico, análise, discussão e interpretação dos resultados, redação e revisão final do artigo.

\section{Agradecimentos}

À Secretaria Estadual do Desenvolvimento Social (Sedes), à coordenadora do Programa de Atenção à Pessoa Idosa (PAPI), Nilsonete Gonçalves Lucena Ferreira, às coordenadoras locais dos Centros de Referência e Cidadania, pelo engajamento na pesquisa, e à Coordenação de Aperfeiçoamento de Pessoal de Nível Superior (Capes) pelo apoio financeiro.

\section{Referências}

1. Papaléo-Netto M. Gerontologia: a velhice e o envelhecimento em visão globalizada. São Paulo: Atheneu; 2002.

2. Organização Mundial da Saúde (OMS). Envelhecimento ativo: uma política de saúde. Geneva: OMS; 2005.

3. Frank AA, Soares EA. Nutrição no envelhecer. São Paulo: Atheneu; 2004.

4. Monteiro CA, Benício MH, Conde WL, Popkin BM. Shifting obesity trends in Brazil. Eur J Clin Nutr 2000; 54:1-5.

5. Monteiro CA, Moura EC, Conde WL, Popkin BM. Socioeconomic status and obesity in adult populations of developing countries: a review. Bull World Health Organ 2004; 82:940-946.

6. Hope AA, Kumanyika SK, Whitt MC, Shults J. Obesity-related comorbidities in obese African Americans in an outpatient weight loss program. Obes Res 2005; 13:772-779.

7. Allison DB, Zhu SK, Plankey M, Faith MS, Heo M. Differential associations of body mass index and adiposity with all-cause mortality among men in the first and second National Health and Nutrition Examination Surveys (NHANES I and NHANES II) follow-up studies. Int J Obesity 2002; 26:410-416.

8. Heo M, Allison DB, Faith MS, Zhu S, Fontaine KR. Obesity and health-related quality of life: the mediating effects of joint pain and comorbidities. Obes Res 2003; 11:209-216.

9. Fontaine KR, Allison DB. Does intentional weight loss affect mortality rate? Eating Behav 2001; 2:87-95.

10. Marti A, Moreno-Aliaga MJ, Hebebrand J, Martinez JA. Genes, lifestyles and obesity. Int J Obes Relat Metab Disord 2004; 28:S29-S36.

11. Lang A, Froelicher ES. Management of overweight and obesity in adults: behavioral intervention for long-term weight loss and maintenance. Eur J Cardio Nurs 2006; 5:102-114.
12. Lefèvre F, Lefèvre AMC, organizadores. Discurso do sujeito coletivo: um novo enfoque em pesquisa qualitativa (desdobramentos). Caxias do Sul: Educs; 2005.

13. Bourdel-Marchasson I, Traissac T. Place et impact des régimes chez les personnes très agrees. Nutr Clin Metab 2004; 18:224-230.

14. Villareal DT, Apovian CM, Kushner RF, Klein S. Obesity in older adults: technical review and position statement of the American Society for Nutrition and NAASO, The Obesity Society. Obes Res 2005; 13:1849-1863.

15. Nahas MV. Atividade física, saúde e qualidade de vida. Paraná: Midiograf; 2001.

16. Organização Mundial da Saúde (OMS). Prevenção das doenças crônicas não-transmissíveis. Geneva: OMS; 2004

17. Organização Mundial da Saúde (OMS). Dieta, nutrição e prevenção de doenças crônicas não-transmissiveis. Geneva: OMS; 2003.

18. Winiewski T. A importância da caminhada para a manutenção da saúde e melhoria da qualidade de vida para a terceira idade. Anais do $16^{\circ}$ Congresso Internacional de Educação Física. Foz do Iguaçu, Paraná, 13-17 jan 2001. Foz do Iguaçu: FIEP; 2001.

19. Deepak Chopra MD. Corpo sem idade, mente sem fronteiras: a alternativa quântica para o envelhecimento. Rio de Janeiro: Rocco; 1999.

Artigo apresentado em 16/03/2009

Aprovado em 28/04/2009

Versão final apresentada em 30/05/2009 